\title{
Operation and Performance of the ATLAS Level-1 Calorimeter and Level-1 Topological Triggers in Run 2 at the LHC
}

\author{
Sebastian Mario Weber* ${ }^{\dagger}$ \\ Kirchhoff-Institute for Physics (DE) \\ E-mail: sebastian.mario.weberecern.ch
}

In Run 2 at CERN's Large Hadron Collider, the ATLAS detector uses a two-level trigger system to reduce the event rate from the nominal collision rate of $40 \mathrm{MHz}$ to the event storage rate of $1 \mathrm{kHz}$, while preserving interesting physics events. The first step of the trigger system, Level-1, reduces the event rate to $100 \mathrm{kHz}$ within a latency of less than $2.5 \mu \mathrm{s}$. One component of this system is the Level-1 Calorimeter Trigger (L1Calo), which uses coarse-granularity information from the electromagnetic and hadronic calorimeters to identify regions of interest corresponding to electrons, photons, taus, jets, and large amounts of transverse energy and missing transverse energy. In this contribution, we discuss improved features and performance of the L1Calo system in the challenging, high-luminosity conditions provided by the LHC in Run 2. A new dynamic pedestal correction algorithm reduces pile-up effects and the use of variable thresholds and isolation criteria for electromagnetic objects allows for optimal performance both at low and high energies.

As the LHC exceeds its design luminosity, it is becoming even more critical to reduce event rates while preserving interesting physics. A new feature of the ATLAS Run 2 trigger system is the Level-1 Topological Trigger (L1Topo), which performs kinematic and angular selections within a latency of $200 \mathrm{~ns}$. The physics motivation for L1Topo, as well as its implementation and performance in the ATLAS trigger system in Run 2, are discussed.

The European Physical Society Conference on High Energy Physics

5-12 July

Venice, Italy

* Speaker.

${ }^{\dagger}$ on behalf of the ATLAS Collaboration. 


\section{Motivation}

Reliable and efficient triggering is an important prerequisite for the physics program conducted with the ATLAS experiment at the CERN Large Hadron Collider (LHC). During Run 2 the LHC has set new records in terms of centre of mass energy $\sqrt{s}$ and instantaneous luminosity $L$. These improvements come at the price of a more challenging experimental environment. The increasing luminosity and pile-up lead to higher trigger rates. These rates have to be limited to a level acceptable for data acquisition and processing.

To achieve this, the ATLAS Run 1 trigger system mainly had the option to increase the energy thresholds of triggers. However, this increase is undesirable, since it reduces the phase space accessible for physics analysis. For the even harsher conditions of Run 2, upgrades at different stages of the trigger chain were implemented. They all aim to improve the signal efficiency, background rejection and pile-up resilience, to keep the trigger thresholds as low as possible. This contribution focuses on the novel features introduced to the ATLAS Level-1 Calorimeter Trigger, namely the dynamic pedestal correction, the improved isolation and energy thresholds and the Level-1 Topological Trigger.

\section{The LHC and the ATLAS detector}

The LHC [1] is a particle accelerator that collides proton $(p)$ bunches with a centre of mass energy of $13 \mathrm{TeV}$, where each bunch contains around $10^{11}$ particles. The spacing between neighbouring bunches is $25 \mathrm{~ns}$, resulting in a collision frequency of $40 \mathrm{MHz}$. The instantaneous luminosity of the machine has exceeded the design value of $L=10^{34} \mathrm{~cm}^{-2} \mathrm{~s}^{-1}$ by nearly a factor of two, as shown in Figure 1 (a). A consequence of the rising luminosity is an increase in the number of $p$ - $p$ collisions in one bunch crossing (BC), termed in-time pile-up. Figure 1 (b) shows the annual increase of the average pile-up in Run 2. The LHC beams are structured in trains of proton bunches which are separated by gaps of empty bunches. The close proximity of the proton bunches in the train leads to additional pile-up effects in the calorimeters, called out-of-time pile-up. They are discussed in Section 4 [2].

The ATLAS experiment at the LHC is a general purpose particle detector. Its innermost part houses a high precision tracking system that covers the region $|\eta|<2.5$ in pseudorapidity. It is based on silicon pixels and strips, as well as gas-filled straw tube sensors and is immersed in the $2 \mathrm{~T}$ magnetic field of a superconducting solenoid. ATLAS has high granularity sampling calorimeters that extend up to $|\eta|=4.9$ and provide near $4 \pi$ coverage. They are based on Liquid Argon technology (LAr) and scintillating tiles. The outer dimensions of the detector are defined by the muon spectrometer and its air-core toroid magnet system that provides a magnetic field with an average field strength of up to $1 \mathrm{~T}$.

Not all collision events produced by the LHC can be recorded permanently by ATLAS. This is due to the limited detector readout and available storage space. ATLAS employs a two-tier trigger system to reduce the trigger rate and select interesting physics events with high $p_{\mathrm{T}}$ final states from the predominant QCD background. The first level, Level-1, is based on custom built, highly parallelized hardware that reduces the rate to $100 \mathrm{kHz}$. The second level, the High Level Trigger 


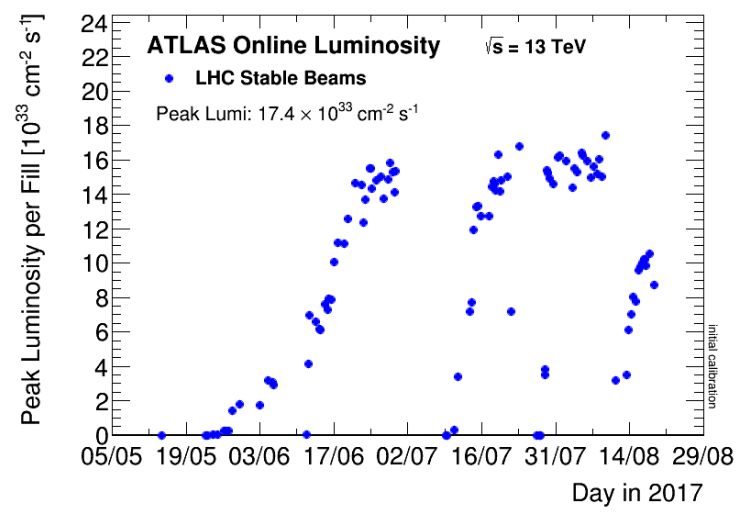

(a)

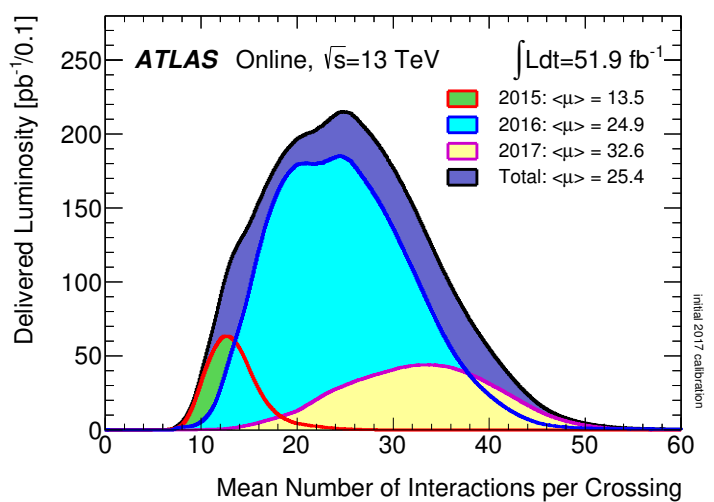

(b)

Figure 1: (a) Peak instantaneous luminosity delivered to ATLAS during stable beam in $p$ - $p$ collisions as a function of time in 2017. (b) Luminosity-weighted distribution of the mean number of interactions per crossing at $13 \mathrm{TeV}$ center-of-mass energy [4].

(HLT), employs a cluster of commercial computers to execute offline-like particle identification algorithms and perform the final trigger selection to decrease the rate to $1 \mathrm{kHz}$ [3].

\section{The ATLAS Level-1 Trigger System}

The ATLAS Level-1 Trigger System is formed by the Level-1 Calorimeter Trigger (L1Calo), the Level-1 Muon Trigger (L1Muon), the Central Trigger Processor (CTP) and the Level-1 Topological Trigger (L1Topo).

L1Calo uses reduced calorimeter information to identify jet, $e / \gamma$ and $\tau$ candidates, as well as missing transverse energy $E_{\mathrm{T}}$ and total energy. It receives analogue signals from the calorimeters, which are the sum of single cell signals in so-called trigger towers. These towers have a typical dimension of $0.1 \times 0.1$ in $\eta \times \phi$.

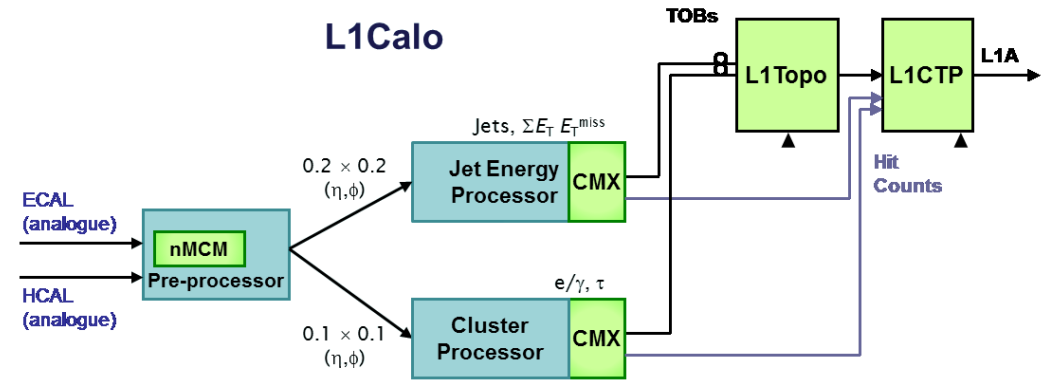

Figure 2: L1Calo architecture during Run 2. Components in green were installed for Run 2 [5].

A schematic overview of L1Calo is given in Figure 2. The initial handling of the trigger tower signals is performed by the PreProcessor ( $\mathrm{PPr}$ ), which treats all trigger towers in parallel. The main components of the PPr are the new Multichip Modules (nMCMs), which are FPGA-based 
and process four trigger towers each. The analogue signals are first synchronized and digitized at $80 \mathrm{MHz}$ with 10 bit precision on the nMCM. The digitized pulses are then sharpened with a finite-impulse response filter and a dynamic correction is applied on the filter output to account for the shifting signal pedestal (c.f. Section 4). The final processing steps on the nMCM are the association of the input signal to the correct LHC collision and the determination of a calibrated $E_{\mathrm{T}}$ value for each trigger tower using a look-up table.

The Cluster Processor (CP) and the Jet/Energy Processor (JEP) use this map of $E_{\mathrm{T}}$ values to identify the electromagnetic and hadronic trigger objects (TOBs) outlined above by means of sliding window algorithms. They contain information about the type, energy and coordinates of the object. Common Merger Modules (CMXs) transmit the TOBs to L1Topo and determine hit counts of the objects that pass a configurable $E_{\mathrm{T}}$ threshold. These hit counts are transmitted to the CTP and are used in the Level-1 Trigger decision.

L1Muon identifies muons with high transverse momentum using dedicated Resistive Plate Chambers and Thin-Gap Chambers in the muon system. It also transmits the information about identified muon TOBs to the CTP and L1Topo (c.f. Section 6) [6].

\section{Dynamic Pedestal Correction}

The dynamic pedestal correction subtracts the average pile-up contribution from the L1Calo input signal. In addition to pile-up from the same BC (in-time) the signal is also affected by pile-up from previous BCs (out-of-time). This is due to the shape of the analogue calorimeter pulses. While the ionisation pulse produced by the drift of the charged particles in the calorimeter has the typical triangular shape, the L1Calo input signals have already undergone shaping in the calorimeter electronics. The signal shown in Figure 3 a) has the typical shape of a LAr input signal. It displays a characteristic bipolar shape, where the peaking time and undershoot of the signal are much longer than the time between two BCs. This means that each signal extends into the following BCs.
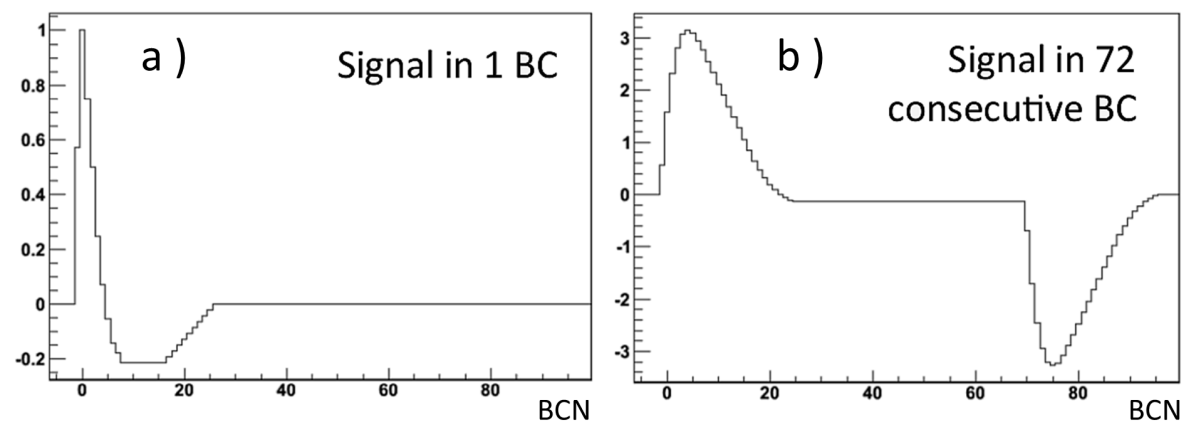

Figure 3: Schematic of LAr pile-up signal for bunch trains of different length as a function of BC number.

A toy study of the resulting pile-up signal as a function of $\mathrm{BC}$ number $(\mathrm{BCN})$ is shown in Figure 3 (b) for a typical bunch train with 72 bunches, each with a signal of the same height. In the middle of the train, the peak of the signal and the undershoot of signals from previous BCs cancel. 


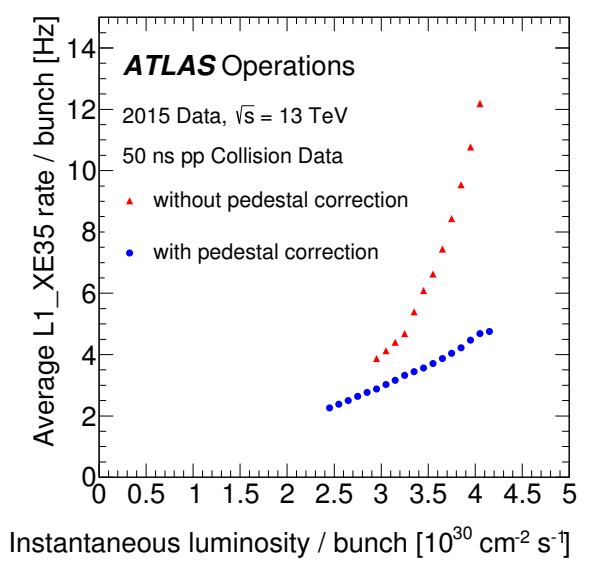

Figure 4: The trigger rate for the missing ET trigger with a threshold at $35 \mathrm{GeV}$ per bunch is plotted as a function of the instantaneous Luminosity per bunch. The rates are shown for different settings with and without pedestal correction applied [7].

Since the train is framed by empty bunches, such a cancellation is not possible at the start and end of the train, leading to a measurable net effect. The height of the pedestal shift is correlated with the exact position of the colliding bunches in the LHC orbit. The signal shape varies in different partitions of the calorimeter which contributes to the $\eta$-dependence of the pile-up signal.

The signal of the pile-up particles is typically small compared to the one produced by the final state particles of the hard interaction. However it is large enough to cause a signal in otherwise empty trigger towers. Therefore the triggers mainly affected by pile-up are those which sum the $E_{\mathrm{T}}$ of many trigger towers, like the sum- and missing $E_{\mathrm{T}}$ triggers. Figure 4 shows the effect of the pile-up contributions on the rate of a missing $E_{\mathrm{T}}$ trigger with a threshold of $35 \mathrm{GeV}$ (L1_XE35, red curve): It rises strongly non-linearly as a function of instantaneous luminosity.

To account for the dependence of the average pile-up on the BCN and the partition of the calorimeter, the pedestal correction is calculated independently for all trigger towers and BCs. Since the pile-up follows the luminosity decrease with time during one fill of the LHC, the correction is recomputed regularly. It is determined by averaging over the filtered signal for $2^{16}$ orbits. Since in most cases the trigger towers have no energy deposition from final state particles of the hard interaction, this gives an accurate measure of the average pile-up contribution. This contribution is then simply subtracted from the filtered signal.

The blue curve in Figure 4 shows the trigger rate of L1_XE35 as a function of luminosity with enabled pedestal correction. The correction reduces the rate considerably and restores a near-linear behaviour. Despite this reduction, the trigger efficiency stays constant [7].

\section{Isolation and variable thresholds at Trigger Level}

The identification of $e / \gamma$ in L1Calo is based on the detection of local energy maxima in the calorimeter using a sliding window algorithm. A sketch of the algorithm is shown in Figure 5 (a).

A central window of $2 \times 2$ trigger towers is moved over the calorimeter and at each position the $E_{\mathrm{T}}$ sum of all possible $2 \times 1$ and $1 \times 2$ trigger tower combinations in the window is calculated. 


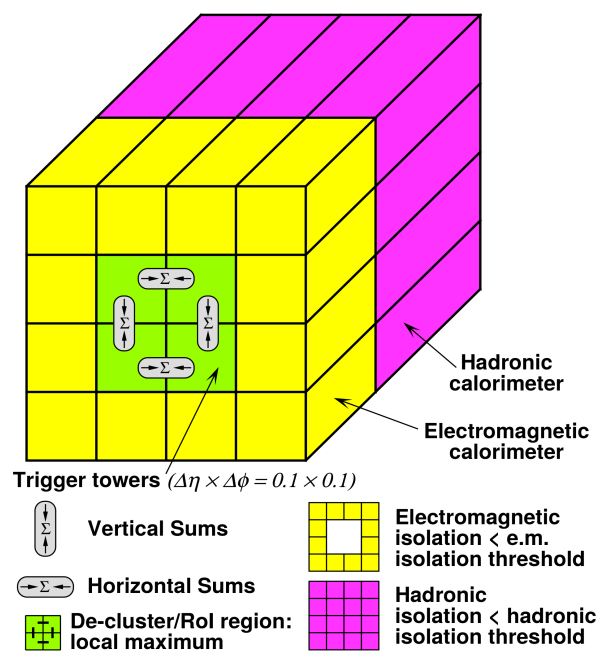

(a)

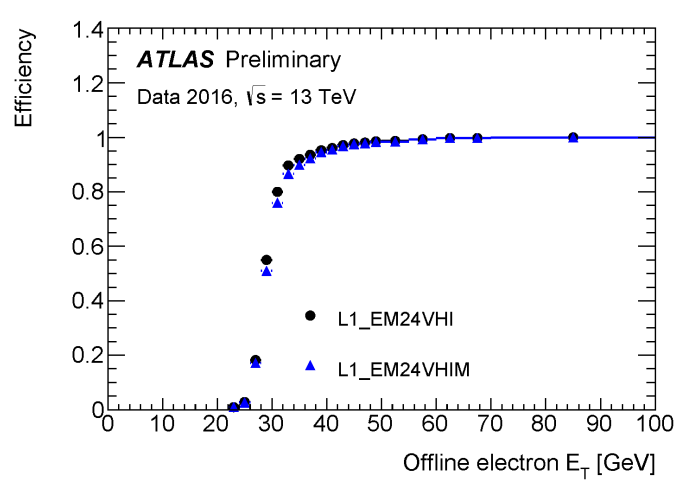

(b)

Figure 5: (a) Elements used for the e/ $\gamma$ and $\tau /$ hadron algorithms [6]. (b) Efficiency of the Level-1 triggers, L1_EM24VHI and L1_EM24VHIM, as a function of the offline electron transverse energy $\left(E_{\mathrm{T}}\right)[8]$.

For a cluster to be identified, one of these sums has to exceed a predefined threshold. For Run 2 this threshold can be chosen variably as a function of $\eta$. This feature is used to account for the varying calorimeter response due to different material budget, geometrical effects and calorimeter technology. The algorithm next compares the direct environment of a cluster to a variable electromagnetic isolation threshold. This isolation threshold is given by

$$
\operatorname{Isol}_{E M}=\max \left(a, b+\frac{E_{\mathrm{T}}}{m}\right)
$$

with $a$ the minimum cut to be used, $m$ the slope of the isolation increase and $b$ the offset that makes it possible to adjust the energy at which the isolation starts to rise above the minimum value. The parameters are re-optimised on a regular basis to account for increasing luminosity. The isolation helps to distinguish electromagnetic showers, as they are usually narrow and fully contained in the $2 \times 2$ core window. The $n M C M$ introduced for Run 2 allows for different energy scales in the electromagnetic and hadronic calorimeter. This feature is used to improve the energy resolution in the electromagnetic calorimeter allowing for finer grained electromagnetic isolation.

The identification algorithm also demands a variable hadronic core isolation, since it is not expected that electromagnetic showers penetrate into the hadronic calorimeter. It requires the energy sum of the hadronic trigger towers directly behind the core cluster and electromagnetic isolation ring to be lower than the value given by

$$
\operatorname{Isol}_{\text {Had }}=\max \left(a, b+\frac{E_{\mathrm{T}}}{m}\right),
$$

where the parameters have the same meaning, but different values, as for the electromagnetic isolation. Triggers using the variable thresholds, hadronic core isolation or electromagnetic isolation are appended by $\mathrm{V}, \mathrm{H}$ and/or I respectively. 
The variable thresholds and the isolation criteria allow for a strong reduction in rate, while keeping the trigger and object efficiency high. The optimization for 2017 (IM) reduced the rate of one of the lowest unprescaled triggers (L1_EM24VHI) by $10 \%$ as compared to 2016 (I). However, the trigger efficiency shown in the right panel of Figure 5 has only changed slightly. Consequently the electron efficiency loss due to the optimized isolation is only around $1 \%[6,8]$.

\section{The ATLAS Level-1 Topological Trigger}

L1Topo was added to the Level-1 Trigger system for Run 2. It is designed to reduce background rates by improving the trigger selection using the topological information of the energy depositions in the calorimeter, like angular separation, invariant mass and hardness of the interaction. To achieve this, L1Topo processes event information in the form of TOBs produced by L1Calo and L1Muon. To allow real-time processing, this information is transmitted to L1Topo over optical links. The topological processor consists of two identical processor blades mounted in an ATCA crate. Each processor blade is equipped with two large FPGAs, which are used to implement in total over 100 algorithms. Different firmware is used on both blades to evenly split these algorithms. The total latency of L1Topo is around $200 \mathrm{~ns}$ per event.

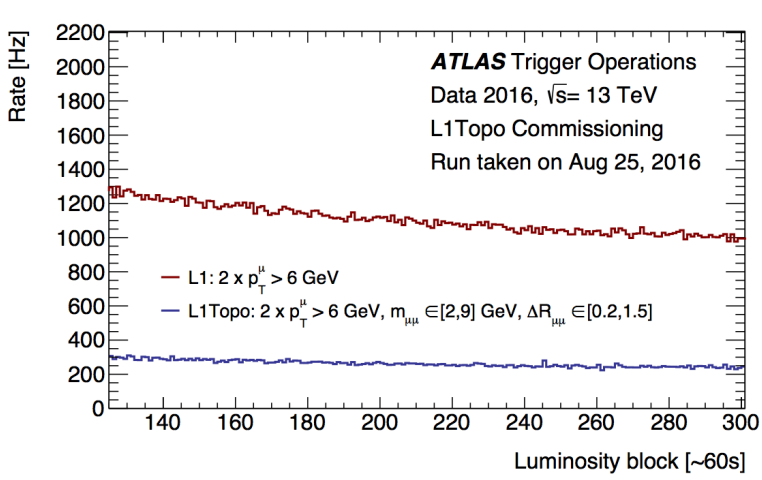

(a)

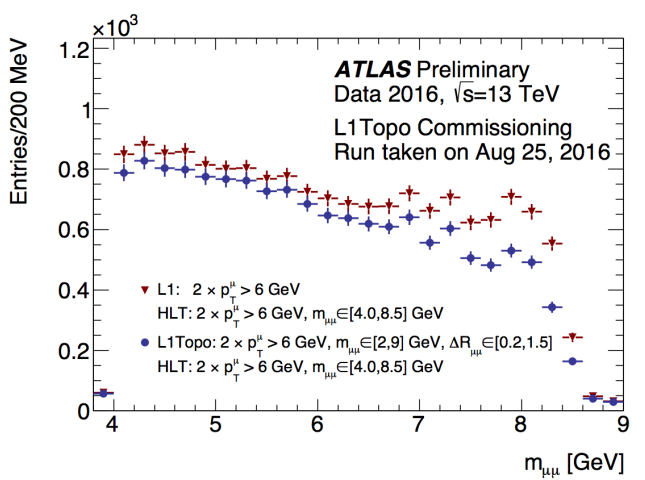

(b)

Figure 6: (a) The rate of Level-1 items that selects two muons, each with transverse momentum above $6 \mathrm{GeV}$ with (red) and without (black) L1Topo additional requirement. (b) The invariant mass distribution for pairs of oppositely charged offline reconstructed muons, shown for events passing a nominal HLT trigger chain (red) and the equivalent HLT chain with additional L1Topo requirements (blue) [9].

L1Topo was shown during comissioning to increase the signal purity of triggered events. Figure 6 (a) shows the rate of a Level-1 dimuon trigger $\left(p_{\mathrm{T}}>6 \mathrm{GeV}\right)$ with and without topological requirements in blue and red respectively. The additional use of cuts on the invariant mass and the angular separation of the dimuon pair reduces the rate by a factor of four. Despite the lower rate at Level-1, the efficiency of the corresponding HLT trigger is only slightly reduced. This can be inferred from Figure 6 (b) that shows the invariant dimuon mass distribution at HLT level for the events selected by Level-1 with and without the topological requirements $[9,10]$. 


\section{Conclusion}

The ATLAS Level-1 Trigger has been upgraded to account for the more challenging experimental environment in Run 2 of the LHC that is characterized by a rising luminosity beyond design and increasing pile-up. The improvements include a dynamic pedestal correction of the calorimeter input signals to the L1Calo trigger, which reduces fake rates due to pile-up, especially for the missing and sum $E_{\mathrm{T}}$ triggers. Electromagnetic trigger items benefit from variable thresholds and isolation in the $\mathrm{CP}$ system. These optimizations reduce the trigger rate, while preserving the signal efficiency. The Level-1 Topological Trigger is a new system added for Run 2. It uses topological information from the calorimeters and the muon system to improve background rejection. The combination of these additions to the ATLAS trigger system help to guarantee optimal data-taking conditions for physics analyses in Run 2.

\section{References}

[1] Evans, L., Bryant, P., LHC Machine Journal of Instrumentation 3 (2008) S08001

[2] The ATLAS Collaboration, Performance of pile-up mitigation techniques for jets in $\mathrm{p} p$ collisions at $\mathrm{s}$ $=8 \mathrm{TeV}$ using the ATLAS detector, The European Physical Journal C 76 (2016) 581

[3] The ATLAS Collaboration, The ATLAS Experiment at the CERN Large Hadron Collider Journal of Instrumentation 3 (2008) S08003

[4] The ATLAS Collaboration, Public luminosity results, https://twiki.cern.ch/twiki/bin/view/AtlasPublic/LuminosityPublicResultsRun2

[5] The ATLAS Collaboration, Technical Design Report for the Phase-I Upgrade of the ATLAS TDAQ System, ATLAS-TDR-023 (2013)

[6] Achenbach et al., The ATLAS Level-1 Calorimeter Trigger, Journal of Instrumentation 3 (2008) P03001

[7] The ATLAS Collaboration, Performance of the ATLAS trigger system in 2015, The European Physical Journal C 77 (2017) 317

[8] The ATLAS Collaboration, Public Egamma Trigger Plots for Collision Data, https://twiki.cern.ch/twiki/bin/view/AtlasPublic/EgammaTriggerPublicResults

[9] The ATLAS Collaboration, Trigger Operation Public Results, https://twiki.cern.ch/twiki/bin/view/AtlasPublic/TriggerOperationPublicResults

[10] E. Simioni et al., Upgrade of the ATLAS Level-1 Trigger with event topology information, Journal of Physics: Conference Series 664 (2015) 082052 